\title{
THE STABILITY OF A NEAR-ADIABATIC ENDEX BATCH CSTR REACTOR
}

\author{
A. C. MCINTOSH ${ }^{1}$, B. F. GRAY ${ }^{2}$, G. C. WAKE ${ }^{3}$ and R. BALL ${ }^{4}$
}

(Received 10 December, 1998; revised 4 April, 1999)

\begin{abstract}
Many tens of serious incidents involving reactors occur in the developed countries each year. The disaster at the chemical plant in Bhopal, India in 1984 was particularly notable where a thermal runaway process led to more than 3000 tragic fatalities from the cloud of extremely toxic methyl isocyanate that boiled out of a storage tank. This signalled the design of special types of chemical reactors to reduce the risk of thermal runaway by planning (at the design stage) integral safety and thermal stabilization mechanisms. The Endex CSTR (continuously stirred tank reactor) proposed by Gray and Ball [3] involves a reactor in two parts with heat exchange allowed between them. The two parts of the reactor operate side by side in tandem, such that the thermal runaway of one part is offset by an endothermic reaction in the other reactor-hence the term 'endex'.

It is found that the adiabatic endex system has a large region of parameter space where the operation can be made safe. However adiabatic conditions rely on the continuous supply of reactants to the endothermic side of the reactor, for operation of the system. The risks involved are such that it is always safer to operate batch reactors in a non-adiabatic mode. Thus we consider the limiting case of the approach to adiabatic conditions where although the mathematics produces no oscillatory causes for instability, yet there is a narrow but significant area where the stable solution branch is lost and consequently a persistent and unexpected region of instability in what otherwise appears to be a simple CSTR system.
\end{abstract}

\section{Introduction}

This work considers a special type of chemical reactor developed to reduce the risk of thermal runaway by planning at the design stage, integral safety and thermal stabilization mechanisms. Such an approach has been called for in the aftermath of

\footnotetext{
'Department of Fuel and Energy, University of Leeds, Leeds LS2 9JT, UK.

${ }^{2}$ School of Chemistry, Macquarie University, Sydney, Australia.

${ }^{3}$ Department of Mathematics and Statistics, University of Canterbury, Christchurch, New Zealand.

${ }^{4}$ Department of Theoretical Physics, Research School of Physical Sciences and Engineering, Australian National University, Canberra ACT 0200, Australia.

(C) Australian Mathematical Society 2001, Serial-fee code 0334-2700/01
} 
the disaster that occurred in Bhopal, India in 1984 with many tens of serious incidents occurring in developed countries each year [2]. The Endex CSTR (continuously stirred tank reactor) developed by Gray and Ball [3] involves a reactor in two parts with heat exchange allowed between them (see Figure 1 and Ball [1, p. 66]). An exothermic reactor is placed on one side of the system, with an endothermic reactor next to it and with further heat transfer to a controlled ambient temperature. Thus the thermal rise of the exothermic side is counteracted by the endothermic reaction on the other side of the reactor - consequently the name 'endex' is used for this system. Some of the stability studies have been done by Gray and Ball in their earlier work [3] and in particular they have shown that the adiabatic endex system can generally be made safe $[1$, p. 80$]$. However adiabatic conditions are difficult to obtain in practice and furthermore there is reliance on the continued efficiency of the endothermic part of the reactor. In that sense it is not a fail-safe mode of operation. More common practice is to operate batch reactors in non-adiabatic conditions, and the purpose of this paper is to consider the stability of a somewhat simplified system (with reactant depletion ignored) near adiabatic conditions. In particular we consider the limiting case of the approach to adiabatic conditions where although the mathematics produces no oscillatory causes for instability, yet there is a narrow but significant area where the stable solution branch is lost and consequently a persistent and unexpected region of instability in what otherwise appears to be a simple CSTR system.

\section{Mathematical model}

Ignoring reactant depletion, the equations for the simple two-dimensional system in dimensional terms are:

$$
\begin{aligned}
& \begin{array}{l}
\text { Exothermic } \\
\text { reaction }
\end{array} V_{1} c_{1} \frac{d T_{1}}{d t^{\prime}}=V_{1}\left(-\Delta H_{1}\right) X_{1} A_{1} e^{-E_{1} / R T_{1}}-L_{\text {ex }}\left(T_{1}-T_{2}\right), \\
& \begin{array}{c}
\text { Endothermic } \\
\text { reaction }
\end{array} \quad V_{2} c_{2} \frac{d T_{2}}{d t^{\prime}}=-V_{2} \Delta H_{2} X_{2} A_{2} e^{-E_{2} / R T_{2}}-L_{\mathrm{ex}}\left(T_{1}-T_{2}\right) \\
& +L_{d}\left(T_{a}-T_{2}\right)
\end{aligned}
$$

where $c_{1}$ and $c_{2}$ are the volumetric heat capacities $\left(\mathrm{J} \mathrm{m}^{-3} \mathrm{~K}^{-1}\right)$ of the two regions with temperatures $T_{1}$ and $T_{2}(K), \Delta H_{1}$ (negative-exothermic) and $\Delta H_{2}$ (positiveendothermic) are the corresponding reaction enthalpies $\left(\mathrm{J} \mathrm{mol}^{-1}\right)$. Here $V_{1}, V_{2}$ are the volumes $\left(\mathrm{m}^{3}\right)$ of the two regions and $E_{1}, E_{2}$ and $A_{1}, A_{2}$ are the activation energies $\left(\mathrm{J} \mathrm{mol}^{-1}\right)$ and reactivities $\left(\mathrm{s}^{-1}\right)$ respectively of the two reactions with $R$ the Universal Gas constant $\left(8.314 \mathrm{~J} \mathrm{~mol}^{-1} \mathrm{~K}^{-1}\right)$. Also $X_{1}$ and $X_{2}$ are the concentrations (mol.m $\mathrm{m}^{3}$ ) of the reactants (assumed not to be depleting significantly) in the two regions, and $T_{a}$ is 


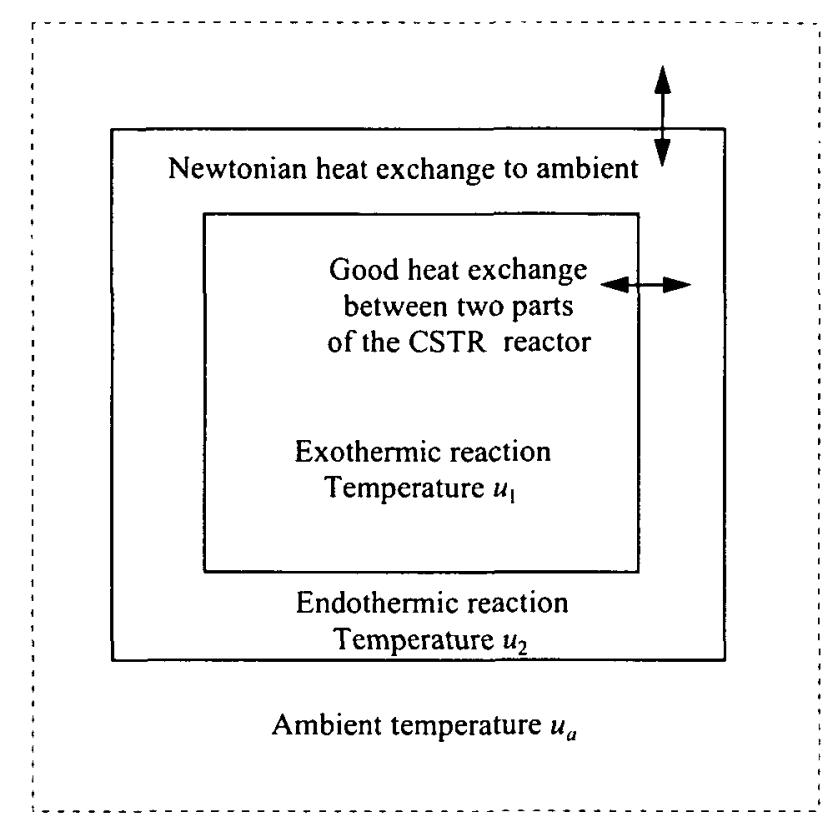

FigurE 1. Schematic of an Endex batch reactor.

the ambient temperature $(\mathrm{K})$ outside the second endothermic part of the reactor. The exchange heat transfer coefficient between the two parts of the reactor $\left(L_{\mathrm{ex}}\right)$ and that between the reactor and the outside $\left(L_{\mathrm{d}}\right)$ are in units of $\mathrm{J} \mathrm{s}^{-1} \mathrm{~K}^{-1}$. Both of these will in fact depend on the relevant exchange surface area at the interchange.

The non-dimensional equivalent of (1) and (2) is

$$
\begin{aligned}
& \frac{d u_{1}}{d t}=e^{-1 / u_{1}}-a\left(u_{1}-u_{2}\right), \\
& \frac{d u_{2}}{d t}=-\lambda e^{-\mu / u_{2}}+b\left(u_{1}-u_{2}\right)+c\left(u_{a}-u_{2}\right),
\end{aligned}
$$

where

$$
\begin{aligned}
& a \equiv \frac{L_{\mathrm{ex}} E_{1} / R}{V_{1} X_{1} A_{1}\left(-\Delta H_{1}\right)}, \quad b \equiv \frac{L_{\mathrm{ex}} E_{1} / R}{V_{1} X_{1} A_{1}\left(-\Delta H_{1}\right)} \frac{c_{1}}{c_{2}}, \\
& c \equiv \frac{L_{\mathrm{d}} E_{1} / R}{\left(-\Delta H_{1}\right) X_{1} A_{1}} \frac{c_{1}}{c_{2}}, \quad \lambda \equiv \frac{\Delta H_{2} X_{2} A_{2}}{V_{1} X_{1} A_{1}\left(-\Delta H_{1}\right)} \frac{c_{1}}{c_{2}}, \quad \mu \equiv \frac{E_{1}}{E_{2}}
\end{aligned}
$$

and the characteristic time scale $\left(c_{1} E_{1} / R\right) /\left(\left(-\Delta H_{1}\right) X_{1} A_{1}\right)$ is used, so that $t \equiv$ $t^{\prime}\left(\left(-\Delta H_{1}\right) X_{1} A_{1}\right) /\left(c_{1} E_{1} / R\right)$. The system becomes effectively the twin system of Gray and Jones [4], but in this paper we have deliberately avoided the Frank-Kamenetskii 
$e^{-E_{1} / R T} \approx e^{-E_{1} / R T_{a}} e^{\phi}$ assumption (where $\phi=E_{1}\left(T-T_{a}\right) /\left(R T_{a}^{2}\right)$, and $E_{1}$ is considered large) used in the earlier work, in order to explicitly expose the ambient temperature as a parameter. In this work we specifically use what are now referred to classically as the 'Gray-Wake' variables where temperature $T$ is non-dimensionalised with respect to the activation temperature $E_{1} / R$ rather than the ambient temperature $T_{a}$. Thus $u_{a}$ is now the non-dimensional representation of ambient temperature. The further advantage of this approach is that there is no loss of information by the exponential assumption. This becomes particularly important when dealing with hysteresis behaviour which in the full system dealt with in this paper, is salient to a very direct formulation of a crucial tongue of instability which appears near adiabatic conditions.

\section{Steady states}

Clearly the steady states of this system are given by the equation set

$$
\begin{aligned}
e^{-1 / u_{1 s}}-a\left(u_{1 s}-u_{2 s}\right) & =0, \\
-\lambda e^{-\mu / u_{2 s}}+b\left(u_{1 s}-u_{2 s}\right)+c\left(u_{a}-u_{2 s}\right) & =0 .
\end{aligned}
$$

The steady state is strictly written here with the subscript 's'. Since virtually all the following analysis is for the steady state, we cease to explicitly use this subscript for ease of notation, from here on. The Jacobian $J$ at the steady state $\left(u_{1}, u_{2}\right)$ is given by

$$
J=\left(\begin{array}{cc}
\frac{e^{-1 / u_{1}}}{u_{1}^{2}}-a & a \\
b & -\lambda \mu \frac{e^{-\mu / u_{2}}}{u_{2}^{2}}-b-c
\end{array}\right),
$$

with the $\operatorname{Tr}(J)$ and $\operatorname{Det}(J)$ governing stability given by

$$
\begin{aligned}
\operatorname{Det}(J) & =-r s-a b, \quad \operatorname{Tr}(J)=r-s ; \\
\text { where } \quad r & \equiv \frac{e^{-1 / u_{1}}}{u_{1}^{2}}-a, \quad s \equiv \lambda \mu \frac{e^{-\mu / u_{2}}}{u_{2}^{2}}+b+c .
\end{aligned}
$$

There could only be oscillatory behaviour in this system if $\operatorname{Tr}(J)=0$ with $\operatorname{Det}(J)>0$, which would imply

$$
r=s \quad \text { with }-r^{2}-a b>0
$$

which is impossible if we have the real situation of energy loss from the exothermic side. Thus there are in fact no Hopf bifurcations in this model. Nevertheless there are some intriguing stability conditions which come out of the hysteresis point analysis.

It is helpful to build up to the details of the full model by going stage by stage, so rather than finding the stability of the full problem immediately, we consider two special cases first. 


\section{Adiabatic case $c=0$}

For the adiabatic case the steady states simplify to

$$
u_{2}=u_{1}-(1 / a) e^{-1 / u_{1}} \quad \text { with } \quad u_{2}=\mu u_{1} /\left(1+d u_{1}\right),
$$

where

$$
d \equiv \ln (a \lambda / b)
$$

is a natural grouped heat transfer parameter linked weakly to the heat exchange coefficients $a$ and $b$, but much more strongly to the enthalpy ratio $\lambda$ of the two reactions.

The steady states of this simpler system are then given by

$$
d=\frac{(\mu-1) a+\left(1 / u_{1}\right) e^{-1 / u_{1}}}{a u_{1}-e^{-1 / u_{1}}} .
$$

For $a=b$ (which from definitions (5) and (6) represents equal heat capacities between the two parts of the reactor), we simply have

$$
d=\ln \lambda .
$$

The steady states for the adiabatic case are illustrated in Figure 2 for the case of the ratio of activation energies $\mu=0.5$ and a heat transfer coefficient $a=0.5$.

Figure 2 serves to demonstrate a typical solution curve for steady states in $u_{1}-d$ space and shows that generally the middle branch is physically relevant, since this acts a watershed for thermal runaway, indicated on the smaller schematics. Thus for practical temperature ranges, one has three solutions or none, similar to the usual $u-u_{a}$ plots of classic ignition theory but with the difference that for any $d$, the lower stable solution is always at $u_{1}=0$. The middle unstable solution is here disconnected from the lower solution $u_{1}=u_{2}=0$ (effectively one can regard it as connected with the lower solution at $d=-\infty$ ).

As can be seen in Figure $2, d=d_{1}$ is a high temperature fold point, so that strictly between $d=d_{1}$ and $d=0$, there is in fact a fourth unstable solution at a very high temperature, but this small region at such high temperatures is not of great physical interest here.

For $a>1 / e$ there are three ranges for $d$. Range (a): For $d<d_{1}$, the endothermicity is very weak. The initial temperature must be very low in order to avoid the unbounded thermal runaway above the watershed steady state curve indicated on the left of Figure 2. Range (b): For $d_{1}<d<d_{2}$, there are three steady-state solutions. These are the safe state $u_{1}=u_{2}=0$, the middle unstable saddle solution, and a high temperature stable solution. The middle unstable solution plotted in Figure 2 is 


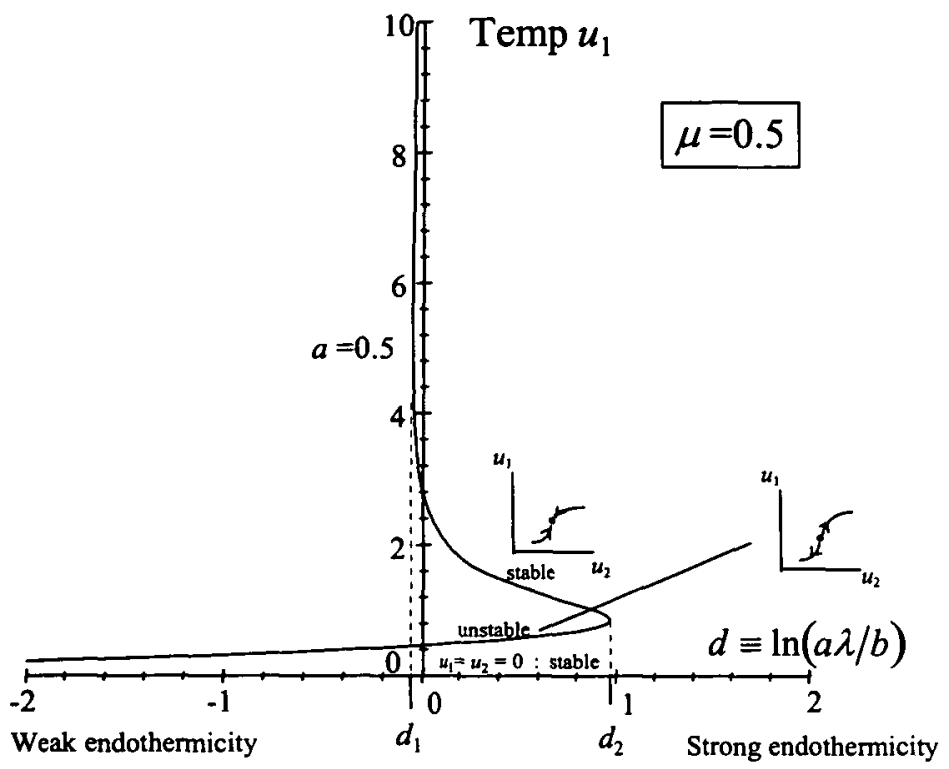

FIGURE 2. Steady states of the adiabatic system.

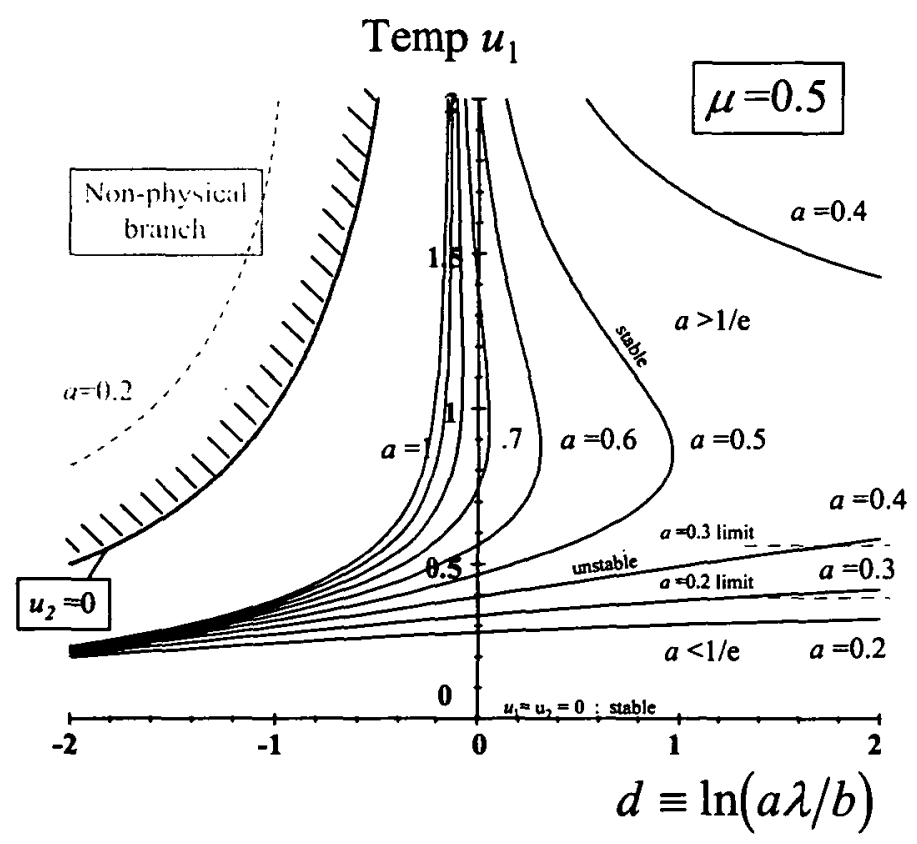

FIGURE 3. Variation with $a$ of steady states of the adiabatic endex reactor system. 


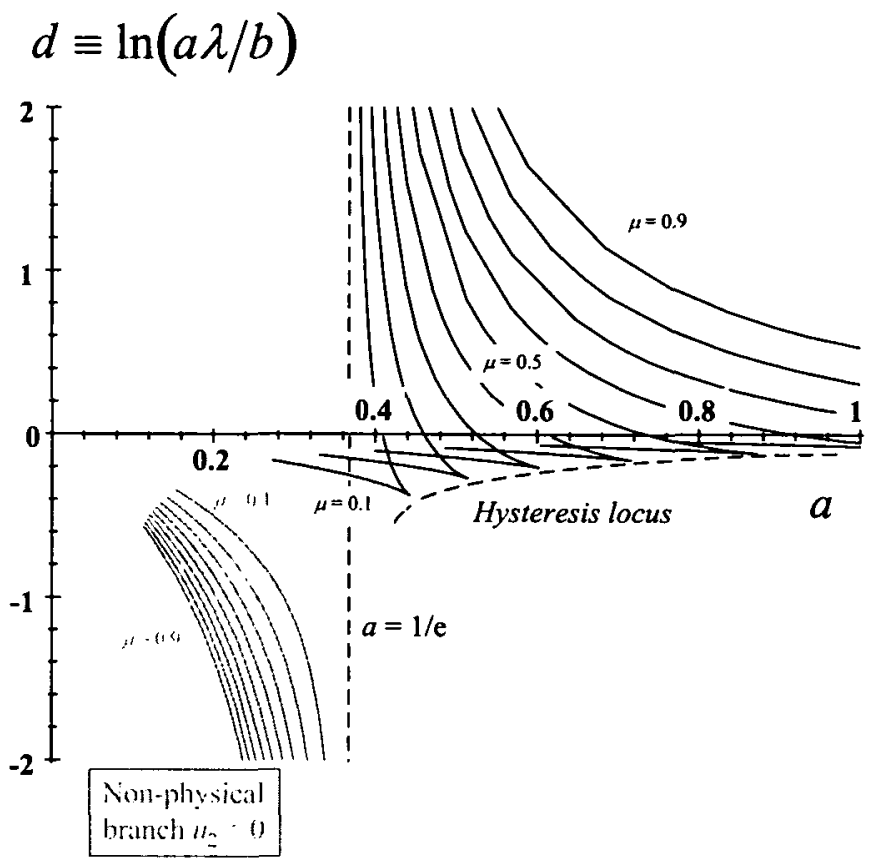

FIGURE 4. Locus of fold points as a function of $d$ and $a$ for a given ratio of activation energies $\mu$.

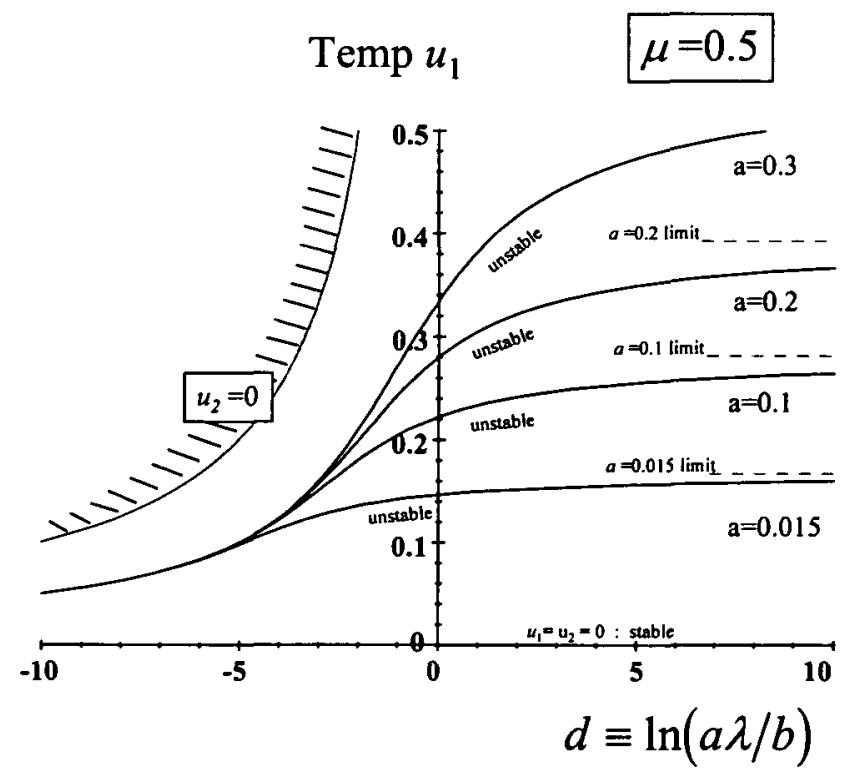

FIGURE 5. Variation with $a$ of steady states of the adiabatic endex reactor system. The heat transfer parameter $a$ is lower than the hysteresis value $1 / e$. Hence one solution only. 
again the important watershed curve which marks out the region below which initial conditions are safe-the endothermicity drives the system to the $u_{1}=u_{2}=0$ safe state. However above this watershed curve, initial conditions are unsafe, since the system will be driven to the dangerous high temperature state. Range (c): For $d>d_{2}$, there is only the lowest steady state $u_{1}=u_{2}=0$ possible. For any initial conditions, the endothermicity is sufficiently high such that the system will always self-cool. In this region the system is always safe. For $a$ greater than a critical hysteresis value $(\approx 0.88$ see Figure 3$)$, then the heat transfer is sufficiently large to lose the fold points all together and beyond the asymptote (at $d$ just less than zero), again we have a self-cooling region.

Figure 3 shows the effect of varying the heat transfer coefficient $a$, with plots of the steady states in $u_{1}-d$ space for $\mu=0.5$.

It can be seen that there is a critical value of $a$ for the adiabatic system

$$
a_{\mathrm{adiab}}^{*}=e^{-1}=0.3679 \ldots
$$

which signals a change in behaviour of the system, such that if $a$ is larger than this value, there is a single $d$ value beyond which there is no steady state.

For $a<1 / e$ (that is, low enough heat transfer), the watershed curve now extends through the whole range of $d$ (even to the strongly endothermic region $d \gg 0$ ), so that one always has the possibility of thermal runaway to an upper very hot stable state, if the initial conditions are hot enough (if not, then the alternative is cooling to the $u_{1}=u_{2}=0$ steady state). This is effectively the lagging effect referred to in the earlier paper of Gray and Jones [4], where the large heat capacity of the outer system acts like a cocoon and there is only weak removal of heat from the exothermic side of the reactor. Consequently de-stabilization occurs if the starting conditions are sufficiently hot, whatever the value of $d$. For a given $a<1 / e$, there is, for that steady state curve, a corresponding limiting $u_{1}$ lim shown dotted on the right of Figure 3 given by the solution to

$$
u_{1 \lim }=\frac{e^{-1 / u_{1 \lim }}}{a}
$$

The locus of the corresponding fold points is indicated in Figure 4 as a function of $d$ and $a$ and for a given ratio of activation energies $\mu$. Thus one of the curves in Figure 3 corresponds to a slice vertically through Figure 4.

This shows that as $a$ is increased, then there is a hysteresis point beyond which the multiplicity of steady states is removed. At the other extreme, as $a$ is decreased below $1 / e$, then (as discussed earlier) the watershed curve extends through all values of $d$ (see Figure 5). 


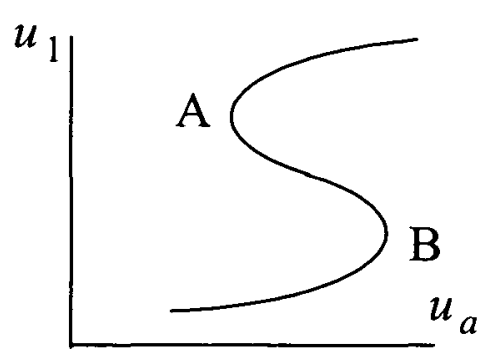

(a)

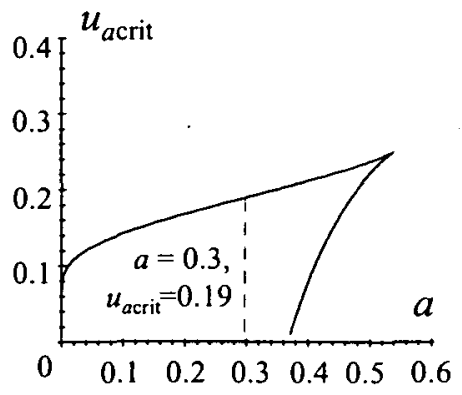

(b)

FIGURE 6. The Semenov limit $c \rightarrow \infty$ (a) typical $u_{1}-u_{a} S$-shaped bifurcation curve and (b) the location of the saddle node (fold) points in $u_{\mathrm{t} \text { crit }}-a$ space.

\section{The Semenov limit $c \rightarrow \infty$}

Before finally coming to the non-adiabatic case, there is one other limit which it is instructive to illustrate - that is the Semenov limit. This is when the heat transfer is excellent at the outer part of the reactor. Consequently for the limit $c \rightarrow \infty$, from (10) and (11) we obtain

$$
\begin{aligned}
& u_{2}=u_{a}, \\
& u_{a}=u_{1}-(1 / a) e^{-1 / u_{1}} .
\end{aligned}
$$

This system has the usual $S$-shaped bifurcation curve shown in Figure 6 (a) with the lower saddle node (fold) loci $A$ and $B$ tracing the curves shown in Figure 6 (b) in $u_{a \text { crit }}-a$ space and given by the solution to

$$
\begin{aligned}
& u_{a \text { crit }}=u_{1 c}-(1 / a) e^{-1 / u_{\mid c}}, \\
& a u_{1 c}^{2}=e^{-1 / u_{\mathrm{lc}}},
\end{aligned}
$$

that is,

$$
u_{1 c}=\frac{1-\sqrt{1-4 u_{a \text { crit- }}}}{2}, \quad u_{1 c}=\frac{1-\sqrt{1-4 u_{a \text { crit }}}}{2}
$$

At the hysteresis point (the cusp in Figure 6 (b)), then we have

$$
a_{\text {hyst }}=4 / e^{2}, \quad u_{a \text { hyst }}=1 / 4 .
$$




\section{Non-adiabatic conditions $c \neq 0$ : Steady states}

For non-adiabatic conditions the steady state equations (10), (11) imply a rather different single equation in $u_{1}$ :

$$
u_{a}=u_{1}-\frac{e^{-1 / u_{1}}}{a}-\frac{b e^{-1 / u_{1}}}{a c}+\frac{\lambda}{c} \exp \left(\frac{-\mu}{u_{1}-(1 / a) e^{-1 / u_{1}}}\right),
$$

and the equation for $u_{2}$ follows on as a separate equation:

$$
u_{2}=u_{1}-(1 / a) e^{-1 / u_{1}} .
$$

The form of the main steady state equation (32) is very different to the corresponding adiabatic version (21) obtained for the adiabatic case. Nevertheless the asymptote for the adiabatic case (24) is clearly still an important asymptote for the non-adiabatic case and is repeated here:

$$
u_{1 \lim }=(1 / a) e^{-1 / u_{1 \mathrm{lim}}} .
$$

This transcendental relationship is plotted in Figure 7 which shows that there is still a critical value of $a, a^{*}=e^{-1}=0.3679 \ldots$, where the steady state behaviour will alter as in the adiabatic case. For $a=0.3, u_{1 \lim } \approx 0.3$.

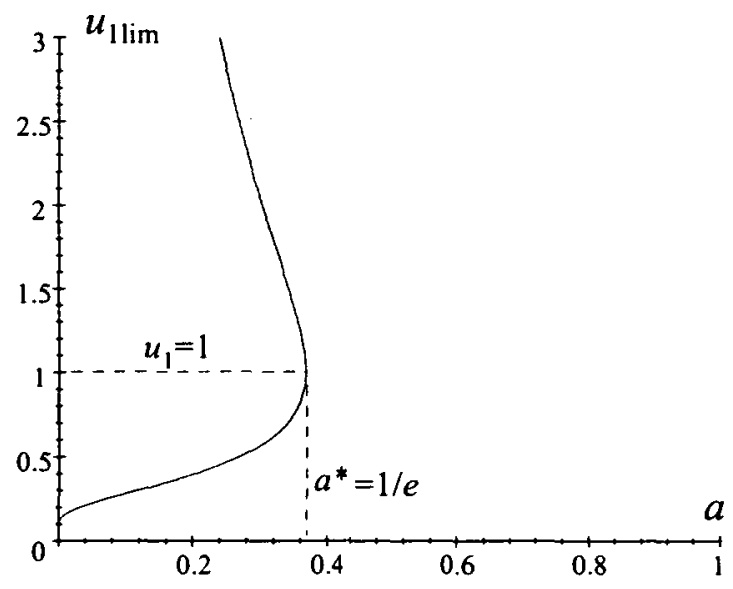

FIGURE 7. Asymptote for steady states of non-adiabatic case.

We now can plot the steady state curves for the full non-adiabatic case with some insight from these adiabatic and Semenov limits. First we plot a typical set of steady state curves for the case $a>1 / e$. These are shown in Figure 8 with $c$ at small values 


\section{Reactor temp $u_{1}$}

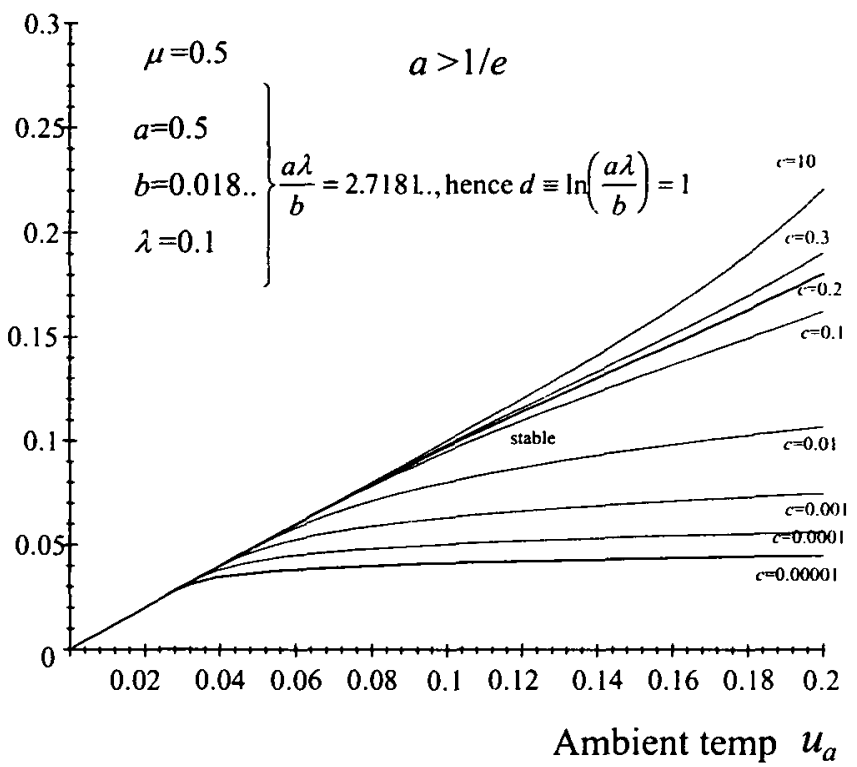

FIGURE 8. Typical steady state curves for near-adiabatic conditions with $a>1 / e$.

\section{Reactor temp $u_{1}$}

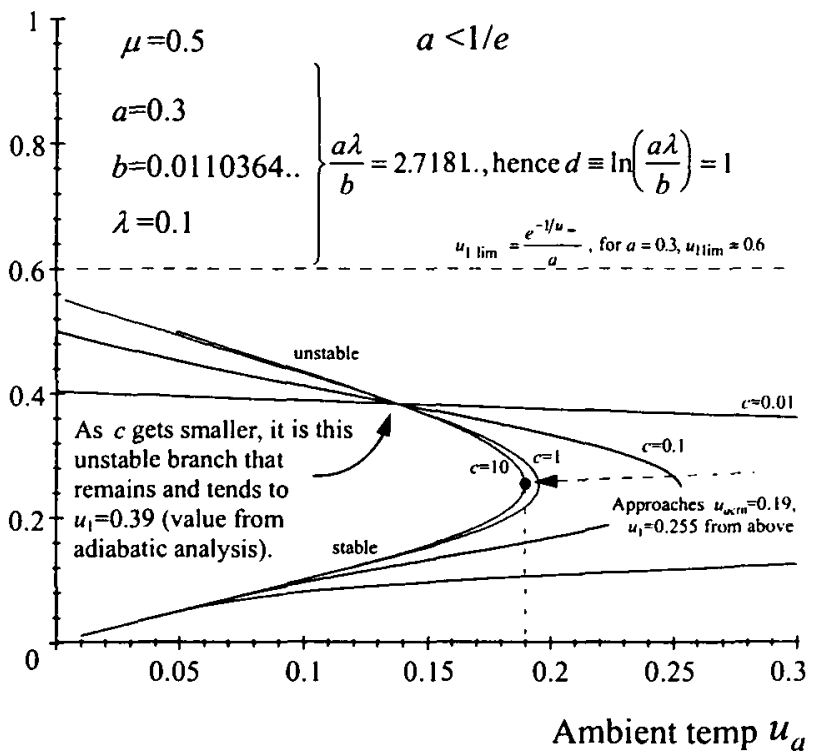

FIGURE 9. Typical steady state curves for near-adiabatic conditions with $a<1 / e$. Approach to Semenov limit with $u_{1}$ from above. 


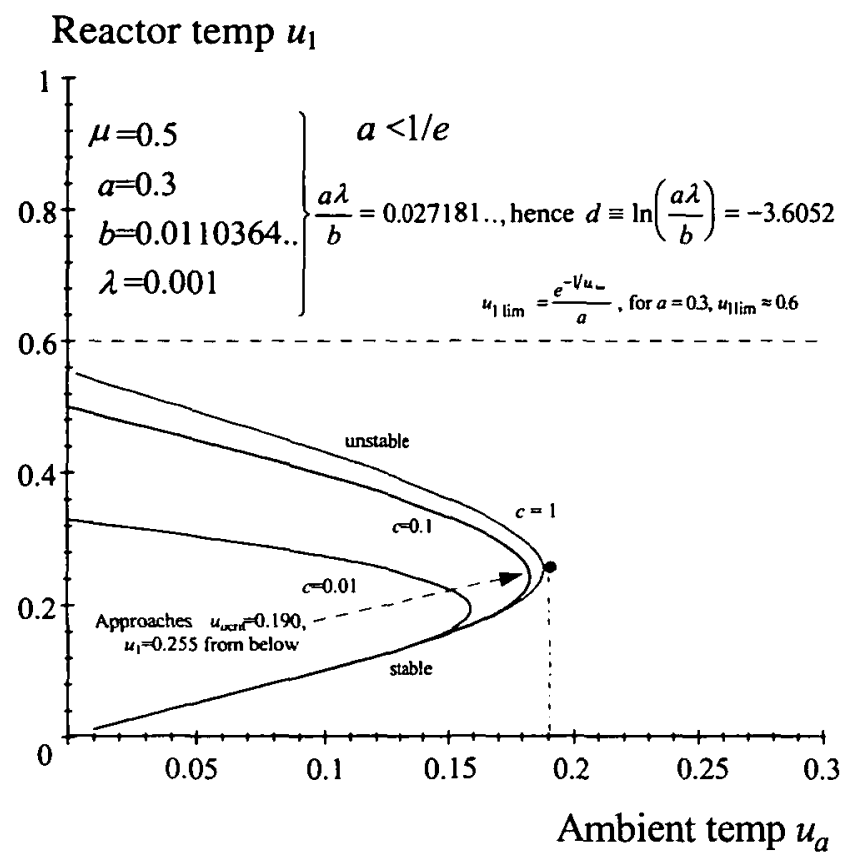

FIGURE 10. Typical steady state curves for near-adiabatic conditions with $a<1 / e$. Approach to Semenov limit with $u_{1}$ from below.

to simulate near-adiabatic conditions. When $a<1 / e$, the plots change their character as is illustrated in Figure 9.

Both these curves illustrate the essential need to let $u_{a}$ be a free parameter to really understand the subtle behaviour that now becomes apparent near adiabatic conditions. The previous work of Gray and Jones [4] was in terms of non-dimensional variables where ambient temperature was tied up in the definition of reactor temperature, so that it could not readily lend itself to a display of the stability criteria for this system.

In Figure 9, as $c \rightarrow \infty$ the Semenov limiting $u_{a \text { crit }}$ (in that case 0.190 ) is approached with $u_{1}$ coming to 0.155 from above. When this approach is from below, then for low values of $c$ (at the other end of the spectrum) there is a small but significant instability near adiabatic conditions. Figure 10 illustrates the steady state curves for $a<1 / e$ but with $\lambda$ changed such that the approach to the Semenov limit is from $u_{1}$ beneath the limiting value, 0.155 .

\section{Non-adiabatic conditions $c \neq 0$ : Stability}

In order to understand the stability of the non-adiabatic system for weak heat losses, we need to track the saddle-node locus because this indicates the region where there 
are no solutions for a given $u_{a}$ greater than the $u_{a \text { crit }}$ corresponding to the fold point. Mathematically, the saddle-node locus is given by where the steady state relationship (25) and its derivative are both zero. Thus we require that $u_{a}=0$ and $d u_{a} / d u_{1}=0$ together, that is,

$$
u_{a}=u_{1}-\frac{e^{-1 / u_{1}}}{a}-\frac{b e^{-1 / u_{1}}}{a c}+\frac{\lambda}{c} \exp \left(\frac{-\mu}{u_{1}-(1 / a) e^{-1 / u_{1}}}\right)
$$

and

$$
\begin{aligned}
1- & \frac{e^{-1 / u_{1}}}{a u_{1}^{2}}-\frac{b e^{-1 / u_{1}}}{a c u_{1}^{2}} \\
& +\frac{\lambda}{c} \exp \left(\frac{-\mu}{u_{1}-(1 / a) e^{-1 / u_{1}}}\right)\left[\frac{-\mu\left(1-e^{-1 / u_{1}} /\left(a u_{1}^{2}\right)\right)}{\left(u_{1}-(1 / a) e^{-1 / u_{1}}\right)^{2}}\right]=0 .
\end{aligned}
$$

At the critical condition, it can be shown that $u_{1 c}$ and $u_{a \text { crit }}$ obey

$$
\begin{gathered}
c\left(1-\frac{e^{-1 / u_{1 c}}}{a u_{1 c}^{2}}\right) \\
=\frac{b e^{-1 / u_{1 c}}}{a u_{1 c}^{2}}-\lambda \exp \left(\frac{-\mu}{u_{1 c}-(1 / a) e^{-1 / u_{1 c}}}\right)\left[\frac{-\mu\left(1-e^{-1 / u_{1 c}} /\left(a u_{1 c}^{2}\right)\right)}{\left(u_{1 c}-(1 / a) e^{-1 / u_{1 c}}\right)^{2}}\right]=0, \\
u_{a c \text { crit }}=u_{1 c}-\frac{e^{-1 / u_{1 c}}}{a}-\frac{b e^{-1 / u_{1 c}}}{a c}+\frac{\lambda}{c} \exp \left(\frac{-\mu}{u_{1 c}-(1 / a) e^{-1 / u_{1 c}}}\right) .
\end{gathered}
$$

The plot of this locus is shown for 2 cases (Figures 11 and 12) whereas in the corresponding steady state curves (Figures 9 and 10) the Semenov limit $c \rightarrow \infty$, $u_{a \text { crit }} \rightarrow 0.190$ approaches from above and from below respectively. What the curves serve to demonstrate is that there is a critical value of $d \equiv \ln (a \lambda / b)$ which we shall term $d_{\text {crit }}$, which is associated with the change in the stability implications of the fold bifurcation curve. For $d$ greater than this $d_{\text {crit }}$ value, there is a behaviour akin to Figure 11 for the fold bifurcation curve, such that as one approaches adiabatic conditions ( $c=0$ ) the stability improves, in that the range of $u_{a \text { crit }}$ where there is a stable region increases. However the converse is true when $d$ is smaller than this $d_{\text {crit }}$ value, which is shown in Figure 12.

For $d$ smaller than $d_{\text {crit }}$, as one approaches adiabatic conditions $(c=0)$ the stability deteriorates, in that the range of $u_{a \text { crit }}$ where there is a stable region decreases. There is a tongue of instability for very low $c$ values as one comes in to the adiabatic limit, meaning that there is considerable danger operating in this region, since this gives a region of instability for lower (and more accessible) temperatures.

Consequently it becomes all important to ascertain what the $d_{\text {crit }}$ value is in terms of the other parameters in the problem. This we address in the next section. 


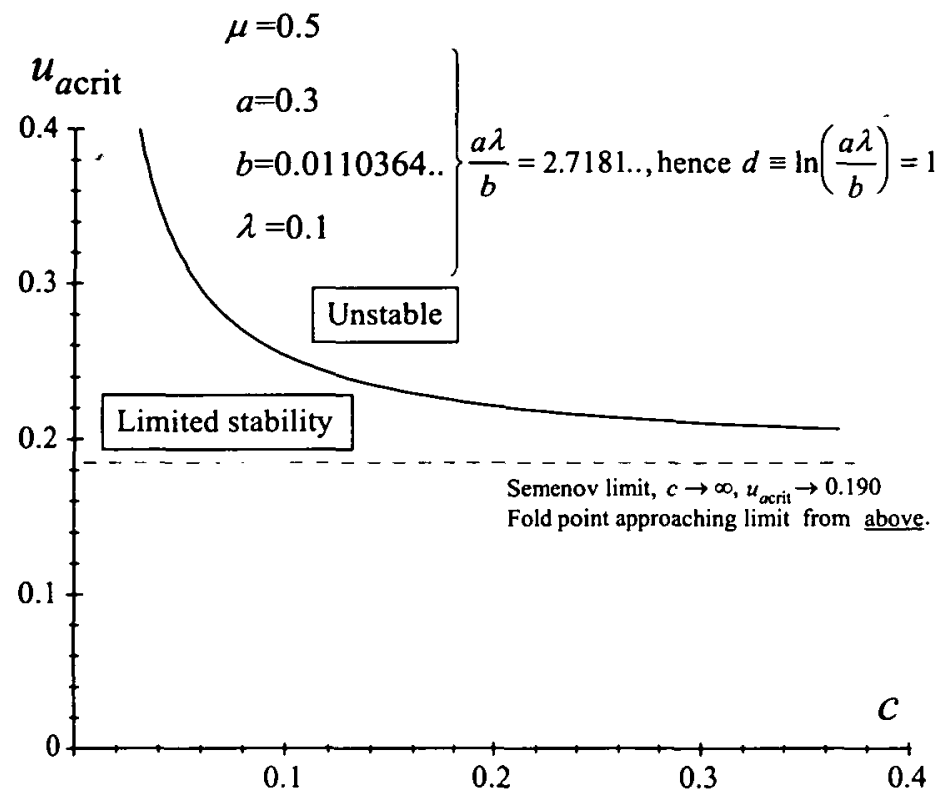

FIGURE 11. Typical saddle-node (fold) locus for non-adiabatic conditions with $a<1 / e$. Approach to Semenov limit with $u_{1}$ from above.

\section{When does the tongue of instability appear near adiabatic conditions?}

In order to address this question we must ask 'when does $u_{a \text { crit }}$ approach $\left.u_{a \text { crit }}\right|_{(c=\infty)}$ from below?' And to answer this we have to return to the Semenov section (Section 5) where we know from (28) that as $c \rightarrow \infty$ we can state approximately

$$
u_{1 c}^{2} \approx(1 / a) e^{-1 / u_{1 c}},
$$

so that following from equation (38) for $u_{a \text { crit }}$, it now follows that for $c \rightarrow \infty$ we can approximately write

$$
\left.u_{a \text { crit }} \approx u_{a \text { crit }}\right|_{c=\infty}-\frac{b}{a c}\left\{e^{-1 / u_{\mathrm{lc}}}-\frac{a \lambda}{b} \exp \left(\frac{-\mu}{\left.u_{a \text { crit }}\right|_{c=\infty}}\right)\right\}
$$

where

$$
\left.u_{a \text { crit }}\right|_{c=\infty}=u_{1 c}-u_{1 c}^{2}, \quad u_{1 c}^{2}=(1 / a) e^{-1 / u_{1 c}}
$$

is the solution for the Semenov limit (see $(27,28)$ of Section 5$)$.

Thus for $u_{a \text { crit }}$ to approach $\left.u_{a \text { crit }}\right|_{(c=\infty)}$ from below (which leads to greater danger), it is necessary to have the second \{bracketed term\} in (40) greater than zero. Thus we 


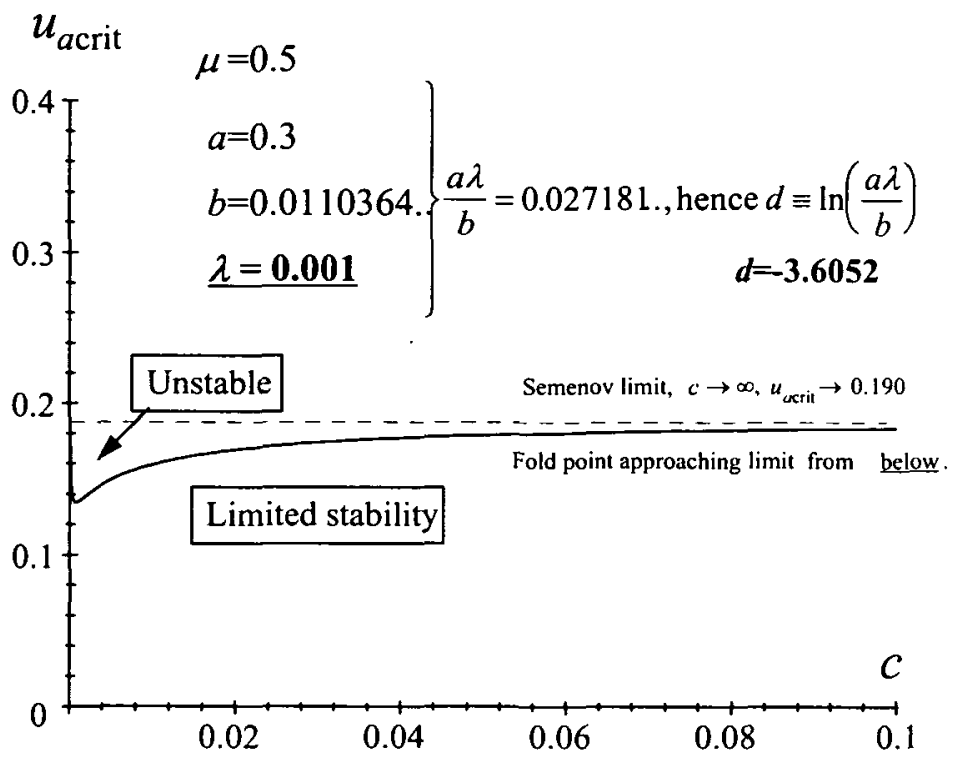

FIGURE 12. Typical saddle-node (fold) locus for non-adiabatic conditions with $a<1 / e$. Approach to Semenov limit with $u_{1}$ from below.

require

$$
e^{-1 / u_{1 c}}-\frac{a \lambda}{b} \exp \left(\frac{-\mu}{\left.u_{a \text { crit }}\right|_{c=\infty}}\right)>0
$$

with

$$
d \equiv \ln (a \lambda / b) \quad \Longrightarrow \quad a \lambda / b=e^{d}
$$

Combining (43b) and (42) gives

$$
\exp \left(\frac{-1}{u_{\mathrm{lc}}}\right)>\exp \left(d-\frac{\mu}{\left.u_{a \text { crit }}\right|_{c=\infty}}\right),
$$

that is,

$$
d<\frac{\mu}{\left.u_{a \text { crit }}\right|_{c=\infty}}-\frac{1}{u_{1 c}} .
$$

Thus the critical value of $d, d_{\text {crit }}$ below which the instability becomes apparent, is given by

$$
d_{\text {crit }} \equiv \frac{\mu}{\left.u_{a \text { crit }}\right|_{c=\infty}}-\frac{1}{u_{1 c}} .
$$




\section{Conclusions}

It is known that adiabatic Endex reactors have large regions of parameter space where safe operation can be obtained [1]. Near adiabatic Endex reactors (which will in practice be the usual mode of operation) have a region of parameter space where a tongue of instability occurs. To avoid this instability, the grouped parameter $d$ (essentially the enthalpy ratio of the endothermic reaction to the exothermic) should be greater than a critical value $d_{\text {crit }}$.

This value of $d_{\text {crit }}$ has been found by recasting the problem first studied by Gray and Jones [4] in variables independent of the ambient temperature and without making the exponential assumption. A clear analytical result then pertains which connects $d_{\text {crit }}$ to the ratio $\mu$ of activation energies and the heat transfer between the two parts of the reactor $a$.

When $d$ becomes less than this critical value then the region of safe $u_{a}$ operation becomes less, and it is well before one reaches adiabatic conditions that the increased danger appears. The critical $d$ is given by

$$
d_{\text {crit }} \equiv \frac{\mu}{\left.u_{a \text { crit }}\right|_{c=\infty}}-\frac{1}{u_{1 c}}
$$

where $\left.u_{a \text { crit }}\right|_{c=\infty}=u_{1 c}-u_{1 c}^{2}$ and $u_{1 c}$ satisfies $u_{1 c}^{2}=(1 / a) e^{-1 / u_{1 c}}$.

A practical example would be for $\mu=0.5, a=0.3,\left.u_{a \text { crit }}\right|_{c=\infty}=0.185$ and $u_{a \text { crit }}=$ 0.245 . Thus $d_{\text {crit }}=0.5 / 0.185-1 / 0.245=-1.379$. For equivalent heat capacities on the two sides of the reactor $(a=b)$ then $\lambda_{\text {crit }} \equiv \exp \left(d_{\text {crit }}\right)=\exp (-1.379)=0.2518$. Thus for $\lambda$ below this value there would be danger. The ratio of endothermic heat of reaction to that for the exothermic heat of reaction must be kept above this value.

It is evident that the behaviour of this tongue of instability will have a crucial effect on the operation of an endex reactor as adiabatic conditions are approached, that is, when $c \rightarrow 0$.

\section{Acknowledgement}

The authors are grateful for helpful and constructive suggestions by Prof. Steve K. Scott of the University of Leeds, on the initial draft of this work.

\section{References}

[1] R. Ball, "Endothermal stabilization of chemical reactors", Ph. D. Thesis, School of Chemistry, Macquarie University, Sydney, Australia, 1996. 
[2] J. A. Barton and P. F. Nolan, "Incidents in the chemical industry due to thermal runaway chemical reactions", in Safety of Chemical Batch reactors and Storage Tanks (eds. A. Benuzzi and J. M. Zaldivar), (ECSC, EEC, EAEC, 1991) 1-17.

[3] B. F. Gray and R. Ball, "Thermal stabilization of chemical reactors: I. The mathematical description of the Endex reactor", Proc. Roy. Soc. A 455 (1999) 163-182.

[4] B. F. Gray and J. C. Jones, "Critical behaviour in chemically reacting systems. IV. Layered media in the Semenov approximation", Comb. and Flame 40 (1981) 37-45. 
